Received 00th January 20xx, Accepted 00th January 20xx

DOI: $10.1039 / \times 0 \times x 00000 x$

\title{
Small molecule anion transporters display in vitro antimicrobial activity against clinically relevant bacterial strains ${ }^{\dagger}$
}

\author{
Israel Carreira-Barral, ${ }^{\mathrm{a}}$ Carlos Rumbo, ${ }^{\mathrm{a}, \mathrm{b}}$ Marcin Mielczarek, ${ }^{\mathrm{a}}$ Daniel Alonso-Carrillo, ${ }^{\mathrm{a}}$ Enara \\ Herran, ${ }^{c}$ Marta Pastor, ${ }^{c}$ Angel Del Pozo, ${ }^{c}$ María García-Valverde ${ }^{a}$ and Roberto Quesada*a
}

Highly active transmembrane anion transporters have demonstrated their activity against antibiotic-resistant and clinically relevant bacterial strains. This type of compounds offers promise as strategy to develop novel antibacterial agents.

The emergence of nosocomial infections caused by multidrugresistant (MDR) bacteria is one of the major threats and challenges of modern medicine. Resistance has been described to nearly all antibiotics currently used in the treatment of infectious diseases. ESKAPE acronym encompasses a group of pathogens that can develop resistance to many of the most used antibiotics and that are frequently involved in hospitalacquired infections. ${ }^{1}$ Enterococcus faecium, Staphylococcus aureus, Klebsiella pneumoniae, Acinetobacter baumannii, Pseudomonas aeruginosa and Enterobacter spp. are included in this group. ESKAPE infections are especially worrying in intensive care units, where these pathogens represent more than $80 \%$ of the infections, posing a great threat to the life of critically ill and immunocompromised patients. ${ }^{2}$ Thus, developing new antimicrobial agents and strategies to combat infections is critical and urgent, both for the treatment of patients and also for disinfecting surfaces and medical devices in hospital environments, where some pathogens can persist even after cleaning, being a significant source for transmission of infection to patients. ${ }^{3}$

Small molecules capable of facilitating membrane permeation to ions, including for instance polyether ionophores and Amphotericin B, constitute relevant examples of widely used antimicrobial agents. ${ }^{4}$ These compounds usually promote unspecific or cation selective membrane permeability. Membrane-permeabilizing agents have also been shown to revert antimicrobial resistance. ${ }^{5-8}$ Membrane disrupting agents such as Polymyxins are used as last resort antimicrobial agents. ${ }^{9}$

\footnotetext{
a. Departamento de Química, Universidad de Burgos, Burgos 09001, Spain. E-mail: rquesada@ubu.es

${ }^{b}$ International Research Centre in Critical Raw Materials-ICCRAM, Universidad de Burgos, Burgos 09001, Spain.

c. Biokeralty Research Institute AIE, Hermanos Lumière 5, 01510 Miñano, Spain.

+ Electronic Supplementary Information (ESI) available: Experimental details, compound characterization data, anion transport and antimicrobial assays. CCDC 1920524. See DOI: 10.1039/x0xx00000x
}

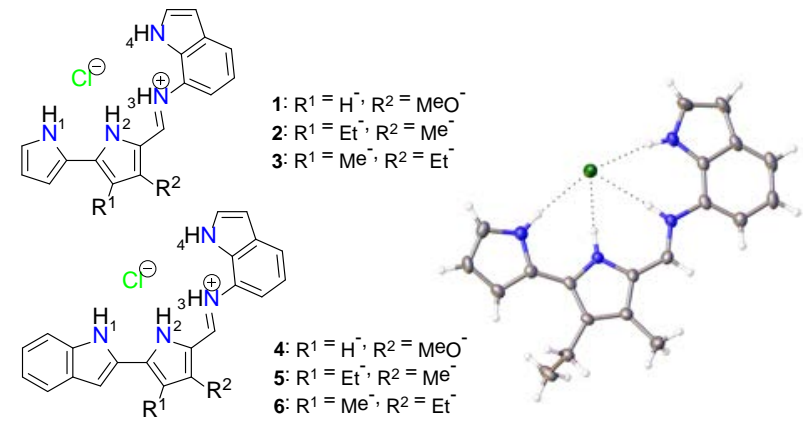

Fig. 1 Compounds 1-6 and solid state X-Ray structure of $\mathbf{2} \bullet \mathrm{HCl}$

The antimicrobial activity of anion selective membrane ionophores, anionophores, ${ }^{10}$ remains much less explored, although encouraging results including activity against methicillin-resistant Staphylococcus aureus (MRSA) have been recently reported. ${ }^{11-13}$ Continuing our efforts to explore the biological activity of anionophores inspired in natural products, ${ }^{14}$ herein we describe the antibacterial activity of indol-7-yl-decorated tambjamine-like compounds against a panel of Gram-positive and Gram-negative bacterial strains as well as clinical isolates, including bacterial strains for which the World Health Organization consider high or critical the urgency of need for new antibiotics. ${ }^{15}$

Compounds 1-6 (Fig. 1) were prepared by condensation of 7-aminoindole and the appropriate aldehyde in refluxing chloroform using acetic acid as catalyst. The compounds were isolated as hydrochloric salts and fully characterized spectroscopically (see ESI for details). These compounds possess a hydrogen bonding cleft defined by four $\mathrm{N}-\mathrm{H}$ groups well suited to interact with anions, as evidenced by data both in the solid state and solution. Slow evaporation of a solution of $2 \cdot \mathrm{HCl}$ in a $1: 1$ chloroform-methanol mixture provided orange single crystals, suitable for X-ray diffraction analysis. In the solid state this compound forms a 1:1 supramolecular complex with the chloride anion. Its skeleton was found essentially flat (torsion angle defined by the four nitrogen atoms $9.66^{\circ}$; mean deviation from the plane formed by the aromatic rings and those from the imine moiety: $0.06 \AA$ ), reflecting high electronic delocalization throughout the aromatic structure (Fig. 1). The four $\mathrm{N}-\mathrm{H}$ fragments of the 
molecule point towards the chloride anion, forming strong hydrogen bonds: the $\mathrm{N} \cdots \mathrm{Cl}$ distances fall in the range 3.09-3.34 $\AA$, whereas the $\mathrm{N}-\mathrm{H}-\mathrm{Cl}$ angles are close to the ideal value $\left(180^{\circ}\right)$.

Titration experiments of hydroperchloric salts of compounds 1-6 in DMSO- $d_{6}$ allowed us to study and quantify anion binding in solution. A representative stack plot of ${ }^{1} \mathrm{H}$ NMR spectra is shown in Fig. 2 and the data were fitted with Bindfit software (Table 1). ${ }^{16}$ In order to determine the association constants for the chloride adducts it is necessary to include a 2:1 ( $\mathrm{LH}: \mathrm{Cl})$ species (LH being the protonated receptor) in the model in all cases, thus suggesting the formation of such supramolecular complexes which then lead to the 1:1 adduct, a situation previously observed in related compounds. ${ }^{17}$ Dilution experiments involving $\mathbf{1} \mathrm{HCl}$ and 1 $\mathrm{HClO}_{4}$ indicated the superior binding affinity of this compound towards chloride compared with perchlorate, consistent with an effective displacement of the latter by chloride in the titration experiments of the perchlorate salts with TBACl. Under the studied conditions, nitrate was found to be weakly coordinating (the displacement of the perchlorate anion is not clearly favoured).

Transmembrane transport experiments were carried out using 1-palmitoyl-2-oleoyl-sn-glycero-3-phosphocoline liposomes (see ESI for details). Chloride release from chloride loaded vesicles was monitored over time with a chlorideselective electrode. ${ }^{18}$ At the end of the experiment, a detergent was added to lyse the liposomes and the resulting chloride reading considered as $100 \%$ release of chloride to normalize the data. In order to assess the relative potency of the carriers, the chloride efflux at 300 seconds for each concentration of compound analysed was plotted against such concentrations and the resulting curve was fitted using Hill analysis. The obtained parameter $E_{50}$ represents the concentration of transporter needed to induce a $50 \%$ chloride release at 300 seconds. The lower the $\mathrm{EC}_{50}$ value, the more potent the anion carrier is (Table 1). Values well below micromolar range were calculated for the $\mathrm{EC}_{50}$ of transporters 1-4, with compounds 5 and 6 displaying a significantly lower

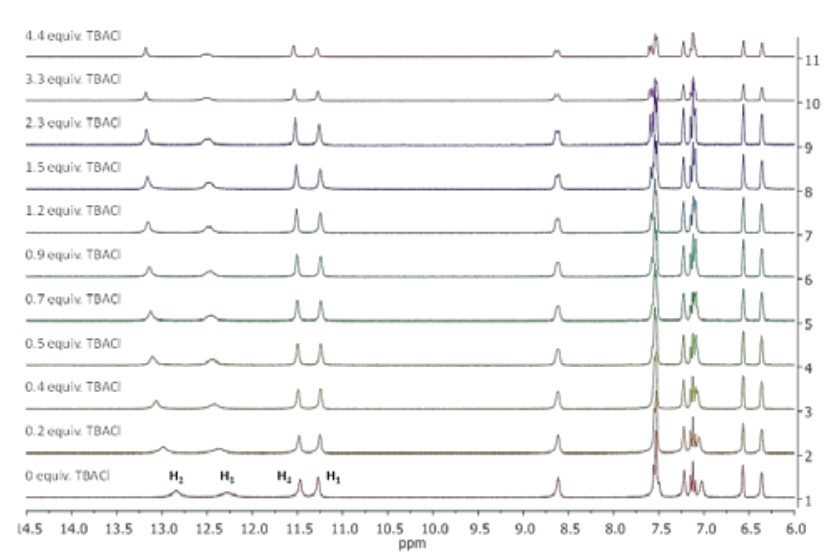

Fig. 2 Stack plot of partial ${ }^{1} \mathrm{H}$ NMR spectra of compound $2 \cdot \mathrm{HClO}_{4}$ in DMSO- $\mathrm{d}_{6}: \mathrm{D}_{2} \mathrm{O}$ with addition of increasing amounts of tetrabutylammonium chloride.
Table 1. Association constants $K_{\mathrm{a}}\left(\mathrm{M}^{-1}\right)$ for compounds 1-6 in their protonated forms (1:1 species) with chloride and nitrate (added as tetrabutylammonium salts), determined from ${ }^{1} \mathrm{H}$ NMR titration experiments in DMSO- $d_{6}$ at $293 \mathrm{~K}$ and transport activities expressed as $\mathrm{EC}_{50}(\mathrm{nM})$.

\begin{tabular}{|c|c|c|c|c|}
\hline Compound & $K_{\mathrm{a}}\left(\mathrm{Cl}^{-}\right)$ & $K_{\mathrm{a}}\left(\mathrm{NO}_{3}{ }^{-}\right)$ & $\begin{array}{c}\mathrm{EC}_{50}(\mathrm{nM}) \\
\mathrm{NO}_{3}{ }^{-} / \mathrm{Cl}^{-}\end{array}$ & $\begin{array}{c}\mathrm{EC}_{50}(\mathrm{nM}) \\
\mathrm{HCO}_{3}{ }^{-} / \mathrm{Cl}^{-}\end{array}$ \\
\hline $\mathbf{1}$ & $549 \pm 39$ & $-{ }^{a}$ & $16 \pm 2$ & $118 \pm 4$ \\
\hline $\mathbf{2}$ & $714 \pm 70$ & $8.8 \pm 0.7$ & $33 \pm 4$ & $896 \pm 79$ \\
\hline $\mathbf{3}$ & $3626 \pm 528$ & $21.5 \pm 4.6$ & $21 \pm 2$ & $666 \pm 103$ \\
\hline $\mathbf{4}$ & $692 \pm 52$ & $13.8 \pm 1.6$ & $27 \pm 2$ & $362 \pm 13$ \\
\hline $\mathbf{5}$ & $834 \pm 71$ & $-{ }^{a}$ & $730 \pm 102$ & $-{ }^{b}$ \\
\hline $\mathbf{6}$ & $1038 \pm 116$ & $1.70 \pm 0.1$ & $1511 \pm 106$ & $-{ }^{b}$ \\
\hline
\end{tabular}

$a$ The association constant value is negligible. ${ }^{b} \mathrm{EC}_{50}$ value could not be determined due to precipitation of the compounds at concentrations above 15 $\mu \mathrm{M}$

activity. These two compounds are more lipophilic than the others studied (see ESI Table S2). Being this parameter central to determine the activity as carriers of this type of compounds, it is possible that their lipophilicity is not optimal to maximize their activity. ${ }^{19}$ The relative variation of EC $_{50}$ when studying chloride efflux in the presence of external nitrate, sulfate or bicarbonate is consistent with anion exchange as the main mechanism accounting for their transmembrane transport activity. Thus, the higher lipophilicity of nitrate compared to that of the other anions makes this anion easier to extract in the lipid membrane and the calculated $E_{50}$ were found smaller in the nitrate/chloride exchange than in the bicarbonate/chloride exchange assay (Table 1).

The antibacterial activity of compounds $\mathbf{1 - 6}$ was first screened on two Gram-positive (vancomycin-resistant $E$. faecium and methicillin-resistant $S$. aureus) and two Gramnegative ( $A$. baumannii and $P$. aeruginosa) bacterial strains. The four of them belong to a group of pathogens with a high rate of antibiotic resistance, being responsible for most of hospital-acquired infections. Experiments to determine the minimum inhibitory concentrations (MICs) were carried out (see ESI for details) and the results are displayed in Table 2 . Overall, the studied compounds were found very effective inhibiting the growth of Gram-positive bacteria, especially $S$. aureus, whereas only compound $\mathbf{1}$ displayed activity against a Gram-negative bacterial strain ( $A$. baumannii). The obtained results correlate well with the transmembrane transport activity of these derivatives. The most potent anionophore 1 was found the most active antibacterial agent whereas the least active anionophore 6 showed the most modest bacterial

Table 2. Minimum inhibitory concentrations (MICs, $\mu \mathrm{M}$ ) for compounds 1-6 against different bacterial strains.

\begin{tabular}{|c|c|c|c|c|}
\hline Compound & E. faecium & S. aureus & A. baumannii & P. aeruginosa \\
\hline $\mathbf{1}$ & 12.5 & 5.21 & 12.5 & $>100$ \\
\hline $\mathbf{2}$ & 16.7 & 8.33 & $>100$ & $>100$ \\
\hline $\mathbf{3}$ & 16.7 & 6.25 & $>100$ & $>100$ \\
\hline $\mathbf{4}$ & 25.0 & 4.16 & $>100$ & $>100$ \\
\hline $\mathbf{5}$ & 25.0 & 8.33 & $>100$ & $>100$ \\
\hline $\mathbf{6}$ & 100 & 16.7 & $>100$ & $>100$ \\
\hline
\end{tabular}


COMMUNICATION

Table 3. Minimum inhibitory concentrations (MICs, $\mu \mathrm{M}$ ) for compound $\mathbf{1}$ for different clinical isolates.

\begin{tabular}{ccccccc}
\hline \multicolumn{7}{c}{ Gram-positive } \\
\hline ATCC Sa29213 & MSSA15 & MSSA16 & MRSA15 & MRSA16 & SE14 & SE94 \\
\hline 3.125 & 3.125 & 3.125 & 3.125 & 3.125 & 3.125 & 1.56 \\
\hline
\end{tabular}

MSSA: methicillin-susceptible Staphylococcus aureus; MRSA: methicillin-resistant Staphylococcus aureus; SE: Staphylococcus epidermidis. ATCC Sa29213 was employed as quality control.

\begin{tabular}{ccccccccccc}
\hline \multicolumn{10}{c}{ Gram-negative } \\
\hline ATCC EC25922 & EC1 & EC2 & EC3 & EC4 & EC5 & KP1 & ATCC Ab19606 & Abl1 & Ab4249 \\
\hline 6.25 & 6.25 & 12.5 & 12.5 & 25 & 6.25 & 12.5 & 6.25 & 3.125 & 3.125 \\
\hline
\end{tabular}

Ec: Escherichia coli; Kp: Klebsiella pneumoniae; Ab: Acinetobacter baumannii. ATCC EC25922 and ATCC Ab19606 were employed as quality controls.

inhibition. Likewise, in agreement with previous reports, the characteristic additional outer membrane of the Gramnegative bacteria represents an effective barrier to prevent the inhibitory action of all compounds except the most active derivative 1 in these bacteria.

The effect of compound 1 on bacterial growth, which presented an inhibitory effect in MIC determinations both in Gram-negative and Gram-positive bacteria, was studied in $A$. baumannii, S. aureus and E. faecium. For all the species, when the growth curve was performed in the presence of 1 at their respective MICs $(12.5,5.21$ and $12.5 \mu \mathrm{M}$ for A. baumannii, $S$. aureus and $E$. faecium, respectively) bacterial growth was totally inhibited (Fig. 3). At concentrations below MICs (3.125 $\mu \mathrm{M})$ a remarkable growth inhibition was observed in $A$. baumannii and S. aureus. Regarding the latter, a delay of $\sim 17$ hours to reach the logarithmic phase with respect to control was noticed, while in $A$. baumannii this delay was $\sim 8$ hours (Fig. 3). In the case of E. faecium, this effect was found less evident, although the cell density reached was lower than that of the control. Overall, these results indicate that compound 1 can interfere with bacterial growth at concentrations below MICs. At the determined MIC, a bactericidal effect against $A$. baumannii was also found (ESI, Figure S116).

In light of the promising antibacterial activity displayed by compound 1, further experiments were carried out in order to assess its activity in both Gram-positive and Gram-negative extensively drug-resistant (XDR) clinical isolates. In the case of Gram-positive bacteria, different methicillin-susceptible and methicillin-resistant $S$. aureus strains along with $S$. epidermidis clinical strains were employed, whereas for Gram-negative 1 was screened towards a variety of $P$. aeruginosa, $E$. coli, $K$. pneumoniae and $A$. baumannii strains. The obtained results confirmed our previous observations. Compound 1 was found effective against all of the tested Gram-positive strains. Moreover, good activity against Gram-negative isolates was also found, particularly A. baumannii, and also $E$. coli and $K$. pneumoniae (Table 3). The MICs determined were found lower for Gram-positive bacteria than Gram-negative, similarly to our previous observations, and no activity was found in the case of $P$. aeruginosa. These results are remarkable since most of these bacteria show a high resistance to many of the most common antibiotics. The fact that $\mathbf{1}$ exhibits broad antibacterial activity suggests that highly active anion transporters could represent a novel class of antimicrobial agents displaying novel modes of action effective against bacteria which are resistant to conventional antibiotics.

We have also studied the interaction of compound $\mathbf{1}$ with blood. Two hemocompatibility tests were performed: a hemolysis and a hemagglutination assays. The levels of hemoglobin released after 1 hour of incubation in the presence of different concentrations of compound $\mathbf{1}$ are displayed in Fig. 4a. Only the highest tested concentration of 1 $(100 \mu \mathrm{M})$ showed significant (more than 10\%) hemolytic activity and at MIC levels hemolytic activity was found to be below 5\%. This percentage, according to ISO/TR/7406, is considered the critical safe hemolytic ratio for biomaterials. ${ }^{20}$ This result is in agreement with a lack of detergent effect exerted by this compound. Hemagglutination assays were then performed. In this assay, the formation of uniform "buttonlike" precipitates after incubation is indicative of hemocompatibility. ${ }^{21}$ Compound $\mathbf{1}$ was serially diluted in

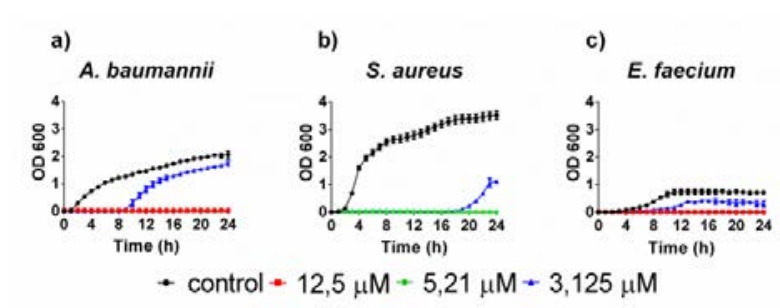

Fig. 3 Growth curves in the presence of compound 1: a) A. baumannii ATCC 17978 incubated with $12.5 \mu \mathrm{M}$ (MIC) and $3.125 \mu \mathrm{M}$ of compound 1; b) S. aureus CECT 5190 incubated with $5.21 \mu \mathrm{M}$ (MIC) and $3.125 \mu \mathrm{M}$ of compound 1; c) E. faecium CECT 5253 incubated with $12.5 \mu \mathrm{M}$ (MIC) and $3.125 \mu \mathrm{M}$ of compound 1 . 
a)

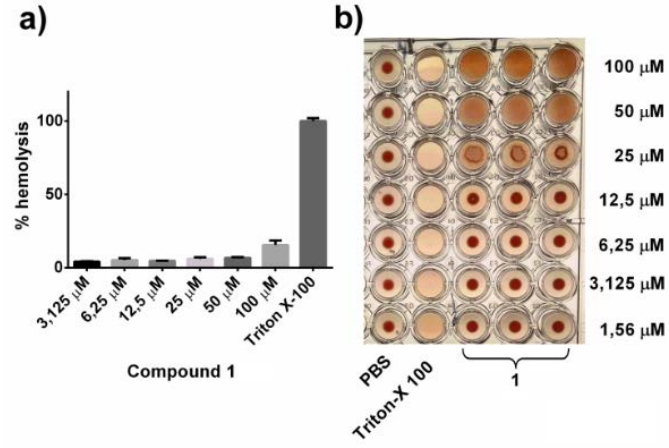

Fig. 4 Hemocompatibility of compound 1: a) hemolysis assay; b) hemagglutination assay.

Dulbecco's Phosphate-Buffered Saline (DPBS) and $100 \mu \mathrm{L}$ of an erythrocyte suspension were added. The results after 12 hours of incubation are shown in Fig. 4b. Little differences with negative control (PBS buffer) can be seen up to $12.5 \mu \mathrm{M}$ concentrations. Addition of higher concentrations of compound $\mathbf{1}$ or the positive control (Triton-X) resulted in altered or no "button-like" precipitate formation. Overall these results indicate that compound $\mathbf{1}$ displays good hemocompatibility at concentrations showing significant antibacterial activity.

In summary, the antibacterial activity of novel indolyldecorated tambjamine analogues bearing different substitutions on the pyrrole ring attached to the imine moiety has been studied. These compounds are highly active transmembrane anion transporters being compound $\mathbf{1}$ the most active compound of the series. Antibacterial studies on bacterial strains and clinical isolates indicate that 1 possesses broad antibacterial activity against both Gram-positive bacteria, like E. faecium and S. aureus, and Gram-negative bacteria, such as $A$. baumannii and $K$. pneumoniae, belonging to a group of pathogens that are frequently involved in hospital-acquired infections. ${ }^{22}$ These results support the potential of highly active transmembrane anion transporters to develop novel antibacterial agents effective against antibiotic-resistant bacteria.

This research has been financially supported by the European Union's Horizon 2020 research and innovation programme (TAT-CF project) under grant agreement $\mathrm{n}$ 667079. The authors gratefully acknowledge Andrea SanchoMedina and Óscar Fernández-Ramos for their contributions to synthesis and transmembrane anion transport experiments, respectively.

\section{Conflicts of interest}

There are no conflicts to declare.

\section{Notes and references}

1 L. B. Rice, J. Infect. Dis., 2008, 197, 1079-1081.

2 G. Zilahi, A. Artigas and I. Martin-Loeches, Ann. Intensive Care, 2016, 6, 96.
3 (a) A. Kramer, I. Schwebke and G. Kampf, BMC Infect. Dis., 2006, 6, 130; (b) P. M. Abreu, P. G. Farias, G. S. Paiva, A. M. Almeida and P. V. Morais, BMC Microbiol., 2014, 14, 118; (c) P. Strassle, K. A. Thom, J. K. Johnsonm, S. Leekha, M. Lissauer, J. Zhu and A. D. Harris, Am. J. Infect. Control, 2012, 40, 1005-1007.

4 (a) M. Kralj, L. Tusek-Bozic and L. Frkanec, ChemMedChem, 2008, 3, 1478-1492; (b) D. S. Palacios, I. Dailey, D. M. Siebert, B. C. Wilcock and M. D. Burke, Proc. Natl. Acad. Sci. U.S.A., 2011, 108, 6733-6738; (c) I. Alfonso and R. Quesada, Chem. Sci., 2013, 4, 3009-3019.

5 M. B. Patel, E. Garrad, J. W. Meisel, S. Negin, M. R. Gokel and G. W. Gokel, RSC Adv., 2019, 9, 2217-2230.

6 J. W. Meisel, M. B. Patel, E. Garrad, R. A. Stanton and G. W. Gokel, J. Am. Chem. Soc., 2016, 138, 10571-10577.

7 S. Negin, M. B. Patel, M. R. Gokel, J. W. Meisel and G. W. Gokel, ChemBioChem, 2016, 17, 2153-2161.

8 (a) S. N. Tyuleva, N. Allen, L. J. White, A. Pépés, H. J. Shepherd, P. J. Saines, R. J. Ellaby, D. P. Mulvihill and J. R. Hiscock, Chem. Commun., 2019, 55, 95-98; (b) S. M. Hickey, T. D. Ashton, G. Boerd, C. A. Bader, M. Thomas, A. G. Elliott, C. Schmuck, H. Y. Yu, J. Li, R. L. Nation, M. A. Cooper, S. E. Plush, D. A. Brooks and F. M. Pfeffer, Eur. J. Med. Chem., 2018, 160, 9-22.

9 T. Velkov, K. D. Roberts, R. L. Nation, P. E. Thompson and J. Li, Future Microbiol., 2013, 8, 711-724.

10 (a) P. A. Gale, J. T. Davis and R. Quesada, Chem. Soc. Rev., 2017, 46, 2497-2519; (b) X. Wu, E. N. W. Howe and P. A. Gale, Acc. Chem. Res., 2018, 51, 1870-1879.

11 A. I. Share, K. Patel, C. Nativi, E. J. Cho, O. Francesconi, N. Busschaert, P. A. Gale, S. Roelens and J. L. Sessler, Chem. Commun., 2016, 52, 7560-7563.

12 C. R. Elie, G. David and A. R. Schmitzer, J. Med. Chem., 2015, 58, 2358-2366.

13 (a) J. Kempf and A. R. Schmitzer, Chem. Eur. J., 2017, 23, 6441-6451; (b) J. Tessier, M. Lecluse, J. Gravel and A. R. Schmitzer, MedChemMed, 2018, 13, 2567-2572.

14 (a) M. Fiore, C. Cossu, V. Capurro, C. Picco, A. Ludovico, M. Mielczarek, I. Carreira-Barral, E. Caci, D. Baroni, R. Quesada and O. Moran, Br. J. Pharmacol., 2019, 176, 1764-1779; (b) C. Cossu, M. Fiore, D. Baroni, V. Capurro, E. Caci, M. GarciaValverde, R. Quesada and O. Moran, Front. Pharmacol., 2018, 9, 852

15 E. Tacconelli, E. Carrara, A. Savoldi, S. Harbarth, M. Mendelson, D. L. Monnet, C. Pulcini, G. Kahlmeter, J. Kluytmans, Y. Carmeli, M. Ouellette, K. Outterson, J. Patel, M. Cavaleri, E. M. Cox, C. R. Houchens, M. L. Grayson, P. Hansen, N. Singh, U. Theuretzbacher and N. Magrini, Lancet Infect. Dis., 2018, 18, 318-327.

16 (a) P. Thordarson, Chem. Soc. Rev., 2011, 40, 1305-1323; (b) (b) www.supramolecular.org

17 L. A. Jowett, E. N. W. Howe, V. Soto-Cerrato, W. V. Rossom, R. Pérez-Tomás and P. A. Gale, Sci. Rep., 2017, 7, 9397.

18 L. A. Jowett and P. A. Gale, Supramol. Chem., 2019, DOI:10.1080/10610278.2019.1574017.

19 N. Busschaert, S. J. Bradberry, M. Wenzel, C. J. E. Haynes, J. R. Hiscock, I. L. Kirby, L. E. Karagiannidis, S. J. Moore, N. J. Wells, J. Herniman, G. J. Langley, P. N. Horton, M. E. Light, I. Marques, P. J. Costa, V. Félix, J. G. Frey and P. A. Gale, Chem. Sci., 2013, 4, 3036-3045.

20 P. R. Sarika, P. R. A. Kumar, D. K. Raj and N. R. James, Carbohydr. Polym., 2015, 119, 118-125.

21 D. Nayak, M. Kumari, S. Rajachandar, S. Ashe, N. C. Thathapudi and B. Nayak, ACS Appl. Mater. Interfaces, 2016, 8, 28538-28553.

22 (a) S. J. Dancer, Clin. Microbiol. Rev., 2014, 27, 665-690; (b) M. P. Muller, C. MacDougall and M. Lim, J. Hosp. Infect., 2016, 92, 7-13. 